\title{
Comparative evaluation of investigations for colorectal carcinoma in symptomatic patients
}

\author{
L. Kalra and A.N. Hamlyn
}

Department of Gastroenterology, Russells Hall and Wordsley Hospitals, Dudley, W. Midlands, UK.

\begin{abstract}
Summary: We studied 154 patients presenting with significant colonic symptoms and subsequently diagnosed to have colorectal carcinoma. They were investigated by faecal occult blood tests, fibresigmoidoscopy, double contrast barium enema (DCBE) and colonoscopy. Faecal occult blood tests (Haemoccult) alone were positive in $26 \%$ of patients with Dukes' $A$, in $69 \%$ with Dukes' B and in $64 \%$ with Dukes' C lesions. DCBE alone identified the lesion in $32 \%$ of Dukes' $A, 79 \%$ of Dukes' $B$ and $81 \%$ of Dukes' $C$ carcinomas. Fibresigmoidoscopy diagnosed colorectal malignancy in $84 \%$ of patients with Dukes' A, 90\% with Dukes' B and $81 \%$ with Dukes' C stage. A diagnostic yield of $88 \%$ for Dukes' A, $96 \%$ for Dukes' $B$ and $100 \%$ for Dukes' $C$ carcinomas was seen with colonoscopy. Detection rate for all stages of carcinoma was greater than $95 \%$ when fibresigmoidoscopy and DCBE were used together. Faecal occult blood tests and DCBE alone are inadequate in diagnosing early malignancy in symptomatic patients. Fibresigmoidoscopy and DCBE used in conjunction compare favourably with the technically difficult procedure of colonoscopy and should routinely be undertaken in these patients before malignancy can confidently be excluded.
\end{abstract}

\section{Introduction}

Colorectal carcinoma is an important cause of death due to malignancy ${ }^{1,2}$ the mortality having remained unchanged for 30 years despite improved surgical techniques. ${ }^{3}$ The only hope for improved survival lies in detecting the lesion before invasion occurs, ${ }^{4}$ hence appropriate investigations in patients presenting with persistent colonic symptoms deserve priority. In this study we have compared relative efficacy of faecal occult blood testing, double contrast barium enema (DCBE), flexible fibresigmoidoscopy and colonoscopy in the diagnosis of colorectal carcinoma.

\section{Methods}

In 1982, a programme aimed at early detection of colorectal carcinoma in symptomatic patients was initiated in the Dudley Health District (estimated catchment population of 296,000). All patients over 40 years of age presenting with significant changes of bowel habit, persistent diarrhoea lasting more than 6 weeks, rectal bleeding, abdominal pain, loss of weight and iron deficiency anaemia were investi-

Correspondence: L. Kalra, M.R.C.P., Queen Mary's Hospital, Sidcup, Kent. DA14 6LT, UK

Accepted: 2 March 1988 gated for malignancy using faecal occult blood tests, flexible sigmoidoscopy and DCBE or colonoscopy. The present study is limited to patients who were diagnosed to have colorectal carcinoma during this programme.

Faecal occult blood was tested by a guaiac slide test (Haemoccult) on 3 consecutive days without any dietary restrictions. Flexible sigmoidoscopy was undertaken either as an outpatient referral or as a direct general practitioner referral (open access). A 60-cm instrument (Olympus OSF, CFP10S) was used with no prior bowel preparation in most patients. These patients were then referred for DCBE or colonoscopy on a random basis. DCBE was undertaken on an outpatient basis after 'Picolax' bowel preparation prior to examination. The radiologist was aware of the patient's history but not of the results of fibresigmoidoscopy. Patients undergoing colonoscopy were given 'Picolax' preparation 1 day prior to the examination and asked to attend the endoscopy unit on the morning of the procedure. Pethidine and midazolam sedation was used and most patients were discharged $2-3 \mathrm{~h}$ after the procedure. Patients in whom fibresigmoidoscopy and DCBE failed to show a lesion were referred for colonoscopy. Similarly, patients with a negative or unsatisfactory colonoscopic examination had DCBE studies. Colonoscopy was considered

(C) The Fellowship of Postgraduate Medicine, 1988 
unsatisfactory if the caecum could not be visualized.

In patients diagnosed to have colorectal malignancy, carcino-embryonic antigen levels were estimated and staging procedures including scintigraphic scans, computerized tomography and laparoscopy were undertaken as appropriate. Patients were staged according to Dukes' classification. Results were analysed for significance by the 'Chi square' test with Yates' correction.

\section{Results}

In all, 154 patients were studied over a period of 5 years. Of these, 81 were men and 73 women with an average age of $66.0 \pm 12.7$ years. The average duration between onset of symptoms and presentation was $6.2 \pm 0.5$ months. Symptoms comprised alteration of bowel habit (114), rectal bleeding (55), loss of weight (53) and iron deficiency anaemia (28).

Dukes' A carcinoma was seen in $40(26 \%)$ patients, 12 of whom were diagnosed on the basis of non-invasion of the polyp stalk on histology following colonoscopic diathermy snare and negative staging procedures. The other 28 patients were staged Dukes' A after surgical resection and histology. Seventy-five $(49 \%)$ patients had Dukes' B carcinoma on the basis of surgical and pathological findings. Dukes' C carcinoma was seen in $39(25 \%)$ patients, 11 of whom were considered inoperable because of metastatic disease identified during staging procedures. Carcinoma was staged as Dukes' C in the remaining 28 patients after surgical histology.

Faecal occult blood tests were undertaken in 95 of the 154 patients. Reliable clinical evidence of rectal bleeding obviated the need for such testing in 55 patients. Testing was unsatisfactory in four patients. Malignancy diagnosed on DCBE examination initiated by the general practitioner obviated the need for further investigations in 28 patients. Fibresigmoidoscopy was performed on 126 patients. Twenty patients were referred for surgery following positive fibresigmoidoscopy. Of the remaining 106 patients, DCBE was undertaken in 65 patients and colonoscopy in 51 patients. Both colonoscopy and DCBE were required in 10 patients, because of negative fibresigmoidoscopy and DCBE in four patients, unsatisfactory colonoscopy in four patients and a negative colonoscopy in two patients.

The overall results of these investigations in the 154 patients studied are given in Table I. Colonoscopy was significantly superior to DCBE in diagnosing all stages of colorectal carcinoma $(P<0.001)$. However, no significant difference in detection rate was seen when colonoscopy was compared to fibresigmoidoscopy and DCBE used in conjunction.

No major adverse complications of fibresigmoidoscopy, DCBE or colonoscopy were recorded in our patients. Minor problems included distension of abdomen, transient abdominal pain and nausea in some patients. Two patients required overnight stay for observations following colonoscopy.

\section{Discussion}

Early detection of colorectal carcinoma is an important consideration in effective management of the disease. Despite increased public awareness in recent years, the vast majority of patients continue to present only when symptomatic, reducing the probability of cure. ${ }^{5,6}$ The objective, therefore, should be to expedite definitive treatment by use of appropriate investigations to confirm or exclude malignancy at an early stage.

Faecal occult blood testing has historically been used as an indicator of colorectal pathology. Large scale screening programmes based on faecal occult blood testing in asymptomatic patients have been undertaken in hope of early diagnosis of colorectal carcinoma $^{7-9}$ but a high failure rate has been recently reported. ${ }^{10}$ The role of faecal occult blood tests in symptomatic patients is even more debatable, being positive in only one-quarter of our patients with a Dukes' A lesion. Faecal occult blood tests are affected by patient compliance, methodology, diet and intermittency of bleeding ${ }^{11}$

Table I Comparison of efficacy of Haemoccult, fibresigmoidoscopy, colonoscopy and DCBE in colorectal carcinoma

\begin{tabular}{lccc}
\hline & \multicolumn{3}{c}{ Dukes' stage } \\
\cline { 2 - 4 } & \multicolumn{1}{c}{$\boldsymbol{A}$} & $\boldsymbol{B}$ & $C$ \\
\hline Haemoccult test & $10 / 38(26 \%)$ & $22 / 32(69 \%)$ & $16 / 25(64 \%)$ \\
Fibresigmoidoscopy & $32 / 38(84 \%)$ & $56 / 62(90 \%)$ & $24 / 26(82 \%)$ \\
DCBE & $10 / 32(32 \%)$ & $33 / 42(79 \%)$ & $19 / 23(81 \%)$ \\
Colonoscopy & $7 / 8(88 \%)$ & $27 / 28(96 \%)$ & $11 / 11(100 \%)$ \\
Fibresig. + DCBE & $38 / 40(95 \%)$ & $74 / 75(99 \%)$ & $38 / 39(98 \%)$ \\
\hline
\end{tabular}


and seldom influence further investigations in symptomatic patients.

DCBE was the cornerstone in diagnosing colorectal carcinoma but its diagnostic accuracy has been recently challenged. ${ }^{12-15}$ In our study, diagnostic yield was disappointingly low in Dukes' A lesions and inadequate cleansing, pre-existing disease or the equivocal nature of early lesions may have reduced accuracy. ${ }^{16}$ However, it continues to be important in diagnosing lesions beyond the reach of a fibresigmoidoscope. ${ }^{17}$

Fifty percent of large bowel neoplasia occur in the distal $25 \mathrm{~cm}$ of the colon. Rigid sigmoidoscopy has poor sensitivity above the distal $16 \mathrm{~cm}$ of the rectosigmoid, hence, is of limited value. Fibresigmoidoscopy, on the other hand, has high sensitivity up to the descending colon and is better tolerated. ${ }^{18}$ Complications are rare and the procedure has been shown to be cost effective. ${ }^{19}$

Colonoscopy is acceptably superior to DCBE in diagnosing colorectal pathology, ${ }^{20}$ but is not widely available at the district general hospital level. It is also a technically difficult procedure with real risks

\section{References}

1. Registrar General's Statistical Review: supplement on cancer 1968-1970. HMSO, London, 1982.

2. CSO Annual abstracts of statistics. HMSO, London 1985.

3. Silverberg, E. \& Holleb, A. Major trends in cancer: 25 year survey. Cancer 1975, 25: 1-8.

4. Bussey, H.J.R. The long term results of surgical treatment of cancer of the rectum. Proc $R$ Soc Med 1963, 56, 494-496.

5. McDermott, F.T., Hughes, E.S.R., Pile, E. et al. Prognosis in relation to symptom duration in colon cancer. Br J Surg 1981, 68: 846-849.

6. Schillaci, A., Cavallaro, A., Nicolanti, V. et al. The importance of symptom duration in relation to prognosis of carcinoma of the large intestine. Surg Gynec Obstet 1984, 158: 423-426.

7. Winawer, S.J., Leidner, S.D., Miller, D.G. et al. Results of a screening program for the detection of early colon cancer and polyps using fecal occult blood testing. Gastroenterology 1977, 72: A 127/1150.

8. Kurnick, J.E., Walley, L.B., Jacob, H.H. et al. Colorectal cancer detection in a community hospital screening programme. JAMA 1980, 243: 2056-2060.

9. Hardcastle, J.D., Farrands, P.A., Balfour, T.W. et al. Controlled trial of faecal occult blood testing in detection of colorectal cancer. Lancet 1983, ii: 1-4.

10. Pye, G. Ballantyne, K.C. \& Hardcastle, J.D. Screening of colorectal carcinoma - analysis of 132 tumours. Gut 1987, 28: A 1344.

11. Nichols, S., Koch, E., Lallemund, R.C. et al. Randomised trial of compliance with screening for colorectal carcinoma. $\mathrm{Br}$ Med $J$ 1986, 293: 107-110. of morbidity and even mortality. In our experience, fibresigmoidoscopy and DCBE used in conjunction were as effective as colonoscopy in diagnosing colorectal carcinoma and hence may be more appropriate as initial investigations to diagnose colorectal malignancy. Diagnostic colonoscopy should be reserved for symptomatic patients in whom fibresigmoidoscopy and DCBE examination are negative or in whom a tissue diagnosis of lesions beyond the reach of a fibresigmoidoscope is essential. We have, as yet, not identified a failure to diagnose colorectal carcinoma in symptomatic patients using this approach.

We believe that fibresigmoidoscopy at the time of presentation followed by an early DCBE examination is the most appropriate investigation for detecting early malignancy in patients over 40 years of age presenting with large bowel symptoms. The extent of tumour spread at the time of its removal is an important factor determining survival ${ }^{21}$ and such screening can contribute significantly in realising the cost effective and life saving potential of early diagnosis of colorectal carcinoma.

12. Teague, R.H., Salmon, P.R. \& Read, A.E. Fibreoptic examination of the colon: a review of 255 cases. Gut 1973, 14: 139-142.

13. Swarbrick, E.T., Fevre, D.I., Hunt, R.H. et al. Colonoscopy in unexplained rectal bleeding. $\mathrm{Br}$ Med $\mathrm{J}$ 1978, 2: 1685-1687.

14. Boulos, P.B., Karmanoles, D.G., Salmon, P.R. et al. Is colonoscopy necessary in diverticular disease? Lancet 1984, i: 95-96.

15. Albridge, M.S. \& Sims, A.J.W. Colonoscopy findings in symptomatic patients without X-ray evidence of colonic neoplasm. Lancet 1986, ii: 833-834.

16. Miller, R.E. Detection of colon cancer and the barium enema. JAMA 1974, 230: 1195-1198.

17. Farrands, P.A., Vellacot, K.D., Amar, S.S. et al. Flexible fibreoptic sigmoidoscopy and double contrast barium enema examination in identification of adenomas and carcinoma of the colon. Dis Colon Rectum 1983, 26: 725-727.

18. Winawer, S.J. Prevention screening and early diagnosis of large bowel cancer. In: DeCosse, J.J. (ed) Large Bowel Cancer. Churchill Livingstone, Edinburgh, London, 1981, pp 46-60.

19. Gilbertsen, V.A.S. \& Nelms, J.M. The prevention of invasive cancer of the rectum. Cancer 1978, 41: 1137-1139.

20. Durdey, P., Weston, P.M.T. \& Williams, N.S. Colonoscopy or barium enema as initial investigation of colonic disease. Lancet 1987, ii: 549-557.

21. Gill, P.G. \& Morris, P.J. The survival of patients with colorectal cancer treated in a regional hospital. $\mathrm{Br} J$ Surg 1978, 65: 17-20. 